\title{
Reviving older drugs to deal with anesthesia drug shortages
}

\author{
Jennifer J. Szerb, MD
}

Received: 23 April 2015/Revised: 25 May 2015/Accepted: 16 July 2015/Published online: 4 August 2015

(C) Canadian Anesthesiologists' Society 2015

Anesthesiologists across Canada are still mourning the withdrawal of preservative-free chloroprocaine from the Canadian market in 2012. This withdrawal occurred due to a combination of corporate decisions by its manufacturer as well as a shortage of the raw materials required to produce chloroprocaine. ${ }^{\mathrm{A}}$ Although not approved for intrathecal use in the United States, chloroprocaine is available for use in Europe (Sintetica S.A., Mendrisio, Switzerland; 10 $\mathrm{mg} \cdot \mathrm{mL}^{-1}$ and an ever-increasing number of studies have been undertaken to establish the optimal dose to provide a safe, reliable, and short-lived subarachnoid blockade. ${ }^{1-4}$ Nevertheless, difficulty in obtaining regulatory approval to study intrathecal chloroprocaine has made it very challenging to perform adequately powered randomized clinical trials in the USA and Canada. ${ }^{5}$ Without chloroprocaine, we must consider "revival of old local anesthetics for spinal anesthesia in ambulatory surgery" 6 in order to maximize efficiency for outpatient surgery.

Prilocaine, an intermediate-acting local anesthetic, was first used in $1965,{ }^{7}$ while ropivacaine, a long-acting agent, was released in $1996 .{ }^{8}$ Until now, there has been a lack of studies comparing the intrathecal administration of these two drugs in terms of time to motor block resolution, postanesthesia care unit (PACU) discharge time, speed of onset, time to voiding, or adverse effects. This lack of data has now been addressed. In this issue of the Journal, Aguirre et al. compare hyperbaric $2 \%$ prilocaine $60 \mathrm{mg}$ with $0.4 \%$ plain ropivacaine $12 \mathrm{mg}$

\footnotetext{
J. J. Szerb, MD ( $\square)$

Department of Anesthesia, Pain Management and Perioperative Medicine, QEII Health Sciences Centre, Dalhousie University, Victoria General Site, 10 West Victoria, 1276 South Park St., Halifax, NS B3H 2Y9, Canada

e-mail: szerbj@ns.sympatico.ca
}

for same-day discharge arthroscopic knee surgery. ${ }^{9}$ The primary outcome, median time to motor block resolution, was significantly shorter in the $2 \%$ prilocaine group than in the $0.4 \%$ ropivacaine group (180 $\mathrm{min}$ vs $240 \mathrm{~min}$, respectively; mean difference, $60 \mathrm{~min}$; $95 \%$ confidence interval $[\mathrm{CI}]$ of difference, 23 to 97; $P<0.036$ ). Nevertheless, this did not translate to a difference in PACU discharge time (330 min for prilocaine vs $335 \mathrm{~min}$ for ropivacaine; mean difference, $5 \mathrm{~min}$; $95 \% \mathrm{CI},-25$ to $35 ; P=0.330)$ as patients were required to void prior to discharge. No neurological sequelae were present in either group after follow-up for $48 \mathrm{hr}$.

The question arises: If the average knee arthroscopy lasts 30-45 min, why would an anesthesiologist choose an anesthetic that lasts at least threefold longer than the duration of surgery? Is prilocaine a reasonable alternative to $35-40 \mathrm{mg}$ of intrathecal chloroprocaine which has a reliable motor offset time of 60 min with an ambulation time of $104 \mathrm{~min} ?^{4}$ The answer is perhaps. With a high prevalence of obstructive sleep apnea ${ }^{10}$ and an aging population with many comorbidities, there are strong arguments to continue to provide subarachnoid anesthesia for our patients. ${ }^{11}$ The avoidance of airway manipulation, respiratory depression, and hemodynamic instability favours providing spinal over general anesthesia in a population who may well require prolonged PACU monitoring anyway (by virtue of their comorbidities). Many anesthesiologists would choose to sacrifice the desirability of a short PACU stay and utilize, for example, low-dose hyperbaric bupivacaine, accepting that there is a wide variation in motor offset time and tolerating

\footnotetext{
A http://www.ashp.org/menu/DrugShortages/CurrentShortages/ Bulletin. aspx ?id=849.
} 
a small chance that, if too low a dose is chosen, the block might fail. ${ }^{12}$

The value of Aguirre et al.'s well-conducted clinical trial is to provide the impetus for further refinement of the prilocaine dosage to make it clinically relevant in the ambulatory knee arthroscopy setting. The authors based their choice of prilocaine dosage on work performed by Guntz et al., ${ }^{13}$ where $40 \mathrm{mg}$ of prilocaine provided effective anesthesia in $92 \%$ of patients. On the other hand, Black et al. reported successful knee arthroscopy with only $20 \mathrm{mg}$ of plain prilocaine and $20 \mu \mathrm{g}$ of subarachnoid fentanyl. ${ }^{14}$ In the Black study, $22 \%$ of patients receiving prilocaine had no demonstrable motor weakness, and $75 \%$ of patients had resolution of motor block at one hour. Discharge times were not specifically commented on in this study. There were no cases of conversion to general anesthesia in the prilocaine group itself. Clearly, there is room for other investigators to attempt further refinement of the optimal dose of intrathecal prilocaine for ambulatory knee arthroscopy.

Finally, we should not forget the value of peripheral nerve blockade for patients where general anesthesia is less favourable. Research has shown that combined femoral/ sciatic block is an acceptable alternative to spinal anesthesia for knee arthroscopy with reasonable discharge times. ${ }^{15}$ Of course, the caveat of this approach is the logistics of placing these blocks well ahead of time in either a dedicated block room or a "swing room" so as not to delay the onset of surgery.

In this era of continued anesthesia drug shortages, anesthesiologists need to use their ingenuity, as well as the data from clinical trials, to find reasonable alternatives. In some circumstances, national organizations need to exert pressure on drug companies to emphasize the importance of anesthesia drugs to the health of our citizens. In the meantime, we will continue to revive what is old in an effort to provide both safe and efficient anesthesia care to our patients.

\section{Raviver les vieux médicaments pour combattre les pénuries de médicaments anesthésiques}

Plusieurs anesthésiologistes regrettent encore le retrait de la chloroprocaïne sans agent de conservation du marché canadien en 2012. Le retrait de cet agent est dû à une combinaison de décisions exécutives du fabricant, et de pénurie des matières premières nécessaires à sa production. ${ }^{\mathrm{A}}$ Bien que son utilisation intrathécale ne soit pas approuvée aux États-Unis, la chloroprocaïne est disponible en Europe (Sintetica S.A., Mendrisio, Suisse; $10 \mathrm{mg} \cdot \mathrm{mL}^{-1}$ ) et un nombre toujours croissant d'études ont été menées afin d'établir la dose optimale pour obtenir une rachianesthésie sécuritaire, fiable et de courte durée. ${ }^{1-4} \mathrm{La}$ difficulté à obtenir l'approbation des autorités pour étudier la chloroprocaïne intrathécale a toutefois rendu très ardue la réalisation d'études cliniques randomisées ayant suffisamment de puissance aux États-Unis et au Canada. ${ }^{5}$ Par conséquent, en l'absence de la chloroprocaïne, nous devons envisager de "ranimer d'anciens anesthésiques locaux pour la rachianesthésie en chirurgie ambulatoire ${ }^{6}$ afin d'optimiser l'efficacité des chirurgies d'un jour.

La prilocaïne, un anesthésique local à action intermédiaire, a d'abord été utilisée en $1965,{ }^{7}$ alors que la ropivacaïne, un agent à durée d'action prolongée, a été distribuée pour la première fois en 1996. ${ }^{8}$ Avant aujourd'hui, aucune étude n'a comparé l'administration intrathécale de ces deux agents en ce qui a trait au temps nécessaire à la résolution du bloc moteur, au moment de congé de la salle de réveil, au délai d'action des blocs, au temps jusqu'à la première miction ou aux effets néfastes - mais cette lacune est désormais comblée. Dans ce numéro du Journal, Aguirre et coll. ont comparé l'utilisation de $60 \mathrm{mg}$ de prilocaïne $2 \%$ hyperbare à $12 \mathrm{mg}$ de ropivacaïne $0,4 \%$ pour une arthroscopie du genou réalisée en clinique ambulatoire. ${ }^{9}$ Le critère d'évaluation principal, soit le temps moyen jusqu'à résolution du bloc moteur, était significativement plus court dans le groupe prilocaïne $2 \%$ que dans le groupe ropivacaïne $0,4 \%$ (180 min vs. $240 \mathrm{~min}$, respectivement; différence moyenne, 60 min; intervalle de confiance [IC] $95 \%$ de différence, 23 à 97; $P<0,036)$. Cependant, cette réduction de temps jusqu'à la résolution du bloc ne s'est pas traduite en une différence en matière de temps jusqu'au congé de la salle de réveil (330 min pour la prilocaïne vs. $335 \mathrm{~min}$ pour la ropivacaïne; différence moyenne, $5 \mathrm{~min}$; IC $95 \%,-25$ à 35; $P=0,330$ ) étant donné qu'on attendait la première miction avant d'accorder le congé aux patients. Aucune séquelle neurologique n'a été observée dans l'un ou l'autre groupe au suivi de $48 \mathrm{~h}$.

Dès lors, la question suivante se pose : si une arthroscopie du genou dure en moyenne 30 à 45 minutes, pourquoi l'anesthésiologiste choisirait-il un agent anesthésique qui agit pendant au moins trois fois plus de temps que la durée de la chirurgie? La prilocaïne constitue-t-elle une alternative acceptable à $35-40 \mathrm{mg}$ de chloroprocaïne intrathécale, dont le temps de récupération d'une fonction motrice stable est de 60 minutes et le temps jusqu'à ambulation de 104 minutes? ${ }^{4}$ La réponse? Peut-être. Étant donné la prévalence élevée d'apnée obstructive du sommeil et une population vieillissante sujette à de nombreuses comorbidités, plusieurs arguments 
justifient de continuer à offrir la rachianesthésie à nos patients. ${ }^{11}$ Chez une population de patients qui pourrait nécessiter un monitorage prolongé en salle de réveil de toute façon, en raison de comorbidités, le fait d'éviter de manipuler les voies aériennes, la dépression respiratoire et l'instabilité hémodynamique pourraient justifier la préférence d'une rachianesthésie plutôt que d'une anesthésie générale. Bon nombre d'anesthésiologistes choisiront de sacrifier un court séjour en salle de réveil et d'utiliser, par exemple, de la bupivacaïne hyperbare à faible dose, acceptant ainsi l'importante variation en matière de temps de récupération de fonction motrice, et tolérant un faible risque d'échec du bloc, si la dose est trop faible. $^{12}$

La valeur de l'étude clinique rigoureuse d'Aguirre et coll. réside dans le fait qu'elle procure l'élan nécessaire pour raffiner la posologie de la prilocaïne afin de la rendre pertinente d'un point de vue clinique dans le contexte d'une arthroscopie du genou réalisée en chirurgie ambulatoire. Les auteurs ont basé leur choix de posologie sur les travaux de Guntz et coll., ${ }^{13}$ dans lesquels $40 \mathrm{mg}$ de prilocaïne ont procuré une anesthésie efficace chez $92 \%$ des patients. Black et coll., en revanche, ont rapporté une arthroscopie du genou réussie avec seulement $20 \mathrm{mg}$ de prilocaïne et $20 \mu \mathrm{g}$ de fentanyl en injection rachidienne. ${ }^{14}$ Dans l'étude de Black, $22 \%$ des patients recevant de la prilocaïne n'ont pas démontré de faiblesse motrice, et le bloc moteur s'est résolu après une heure chez $75 \%$ des patients. Les temps de congé n'ont pas été spécifiquement rapportés dans cette étude. Aucun cas de conversion à l'anesthésie générale n'a été rapporté pour le groupe prilocaïne. D'autres chercheurs peuvent donc assurément tenter de déterminer encore plus précisément la dose optimale de prilocaïne intrathécale pour l'arthroscopie du genou en chirurgie ambulatoire.

Enfin, n'oublions pas l'importance des blocs nerveux périphériques pour les patients chez lesquels l'anesthésie générale n'est pas recommandée. Les recherches démontrent qu'un bloc fémoral / sciatique combiné constitue une alternative acceptable à la rachianesthésie pour l'arthroscopie du genou et procure des temps de congé acceptables. ${ }^{15}$ Bien entendu, l'inconvénient de cette approche réside dans la logistique nécessaire à mettre ces blocs en place bien avant, soit dans une salle dédiée au bloc ou dans une salle « transitoire », afin de ne pas retarder le début de la chirurgie.

À une époque où les pénuries de médicaments anesthésiques sont fréquentes, les anesthésiologistes doivent faire preuve d'ingéniosité, et se servir des données des études cliniques, afin de trouver des alternatives acceptables. Dans certains cas, les organismes nationaux doivent faire pression sur les compagnies pharmaceutiques afin de souligner l'importance des médicaments anesthésiques pour la santé de nos citoyens. En attendant, nous continuerons de raviver les vieux médicaments pour tenter de procurer des soins anesthésiques à la fois sécuritaires et efficaces à nos patients.

Conflicts of interest None declared.

Conflit d'intérêt Aucun.

\section{References}

1. Sell A, Tein T, Pitkanen M. Spinal 2-chloroprocaine: effective dose for ambulatory surgery. Acta Anaesthesiol Scand 2008; 52 : 695-9.

2. Schmittner MD, Gebhardt V. New Short acting local anaesthetics for spinal anesthesia -well tried substances for ambulatory surgery (German). Anasthesiol Intensivmed Notfallmed Schmerzther 2015; 50: 166-73.

3. Camponovo C. Spinal $1 \%$ 2-chloroprocaine versus general anesthesia for ultra-short outpatient procedures: a retrospective analysis. Acta Biomed 2014; 85: 265-8.

4. Kouri ME, Kopacz DJ. Spinal 2-chloroprocaine: a comparison with lidocaine in volunteers. Anesth Analg 2004; 98: 75-80.

5. Pollock J. Intrathecal chloroprocaine - not yet "safe" by US FDA parameters Int Anesthesiol Clin 2012; 50: 93-100.

6. Forster JG, Rosenberg PH. Revival of old local anesthetics for spinal anesthesia in ambulatory surgery. Curr Opin Anaesthesiol 2011; 24: 633-7.

7. Crankshaw TP. Citanest (prilocaine) in spinal analgesia. Acta Anaesthesiol Scand Suppl 1965; 16: 287-90.

8. Zink W, Graf BM. Benefit-risk assessment of ropivacaine in the management of postoperative pain. Drug Saf 2004; 27: 1093-114.

9. Aguirre J, Borgeat A, Buhler P, Mrdjen J, Hardmeier B, Bonvini $J M$. Intrathecal hyperbaric prilocaine $2 \%$ versus plain ropivacaine $0.4 \%$ for same-day arthroscopic knee surgery: a prospective, randomized, double-blind, controlled study. Can J Anesth 2015; 62: this issue. DOI:10.1007/s12630-015-0445-5.

10. Chung SA, Yuan H, Chung F. A systemic review of obstructive sleep apnea and its implications for anesthesiologists. Anesth Analg 2008; 107: 1543-63.

11. Guay J, Choi P, Suresh S, Albert N, Kopps S, Pace NL. Neuraxial blockade for the prevention of postoperative mortality and major morbidity: an overview of Cochrane systematic reviews. Cochrane Datebase Syst Rev 2014; 1: CD010108.

12. Nair GS, Abrishami A, Lermitte J, Chung F. Systematic review of spinal anaesthesia using bupivacaine for ambulatory knee arthroscopy. Br J Anaesth 2009; 102: 307-15.

13. Guntz E, Latrech B, Tsiberidis C, Gouwy J, Kapessidou Y. ED50 and ED 90 of intrathecal hyperbaric $2 \%$ prilocaine in ambulatory knee arthroscopy. Can J Anesth 2014; 61: 801-7.

14. Black AS, Newcombe GN, Plummer JL, McLeod DH, Martin DK. Spinal anaesthesia for ambulatory arthroscopic surgery of the knee: a comparison of low-dose prilocaine and fentanyl with bupivacaine and fentanyl. Br J Anaesth 2011; 106: 183-8.

15. Davarci I, Tuzcu K, Karcioglu M, et al. Comparison between ultrasound-guided sciatic-femoral nerve block and unilateral spinal anaesthesia for outpatient knee arthroscopy. J Int Med Res 2013; 41: 1639-47. 